\title{
Perfil hematológico e bioquímico de primatas não humanos (Callithrix penicillata) cativos no Centro Nacional de Primatas no Estado do Pará
}

\author{
Deise de Lima Cardoso ${ }^{1^{*}}$ \\ Sandra de Mamedes Costa ${ }^{1}$ \\ Roberto de Faria Espinheiro ${ }^{1}$ \\ Paulo Henrique Gomes de Castro ${ }^{2}$ \\ Hilma Lúcia Tavares Dias ${ }^{1}$ \\ ${ }^{1}$ Universidade Federal do Pará \\ Laboratório de Reprodução Animal, Instituto de Ciências Biológicas \\ Avenida Augusto Corrêa, 1, CEP 66.075110, Guamá, Belém - PA, Brasil \\ ${ }^{2}$ Centro Nacional de Primatas, Instituto Evandro Chagas/SVS/MS \\ Rodovia BR-316, Km 7, CEP 67.030-000, Ananindeua - PA, Brasil \\ * Autor para correspondência \\ deisecardosomv@yahoo.com.br
}

Submetido em 21/06/2021

Aceito para publicação em 16/11/2021

\section{Resumo}

Os primatas não humanos da espécie Callithrix penicillata atuam como modelos biológicos para estudo de doenças que acometem os seres humanos, por apresentarem características filogenéticas semelhantes a estes, contribuindo para o avanço nas pesquisas biomédicas. Objetivou-se avaliar os parâmetros hematológicos e bioquímicos de primatas da espécie $C$. penicillata criados em cativeiro. Foram realizadas coletas de sangue de 10 machos e 14 fêmeas, todos adultos, mantidos no Centro Nacional de Primatas-CENP, localizado no município de Ananindeua, Pará. As análises hematológicas e bioquímicas foram realizadas por meio da utilização de contadores automáticos e kits bioquímicos, para o estabelecimento de prováveis diferenças entre sexo relacionadas ao perfil hepático (ALT, AST, GGT), perfil renal (creatinina e ureia), perfil lipídico (colesterol e triglicerídeo) e perfil proteico (albumina e proteínas totais). A análise estatística empregada foi a descritiva para obtenção da média, do desvio padrão, e de valores mínimos e máximos com 95\% de confiança. Todos os animais deste estudo se apresentaram saudáveis e os valores hematológicos e bioquímicos para ambos os sexos se assemelham aos de outras espécies do gênero Callithrix, com variações entre machos e fêmeas. Além disso, os dados analisados podem contribuir sobremaneira com o avanço do estado de arte referente a esse assunto para a espécie em questão, principalmente no que concerne aos valores da bioquímica sérica.

Palavras-chave: Cativeiro; Hematologia

\section{Abstract}

Hematological and biochemical profile of captive non-human primates (Callithrix penicillata) at the National Primate Center in the state of Pará. Due to its similar phylogenetic characteristics, the non-human 
primate Callithrix penicillata is used as a biological model to study diseases that affect humans, which contributes to the advancement of biomedical research. The objective of this study was to evaluate the hematological and biochemical parameters of $C$. penicillatta raised in captivity. Blood samples were collected from 10 males and 14 females, all adults, kept at the National Primate Center (CENP) in the city of Ananindeua, Pará. Hematological and biochemical analyses were conducted using automatic counters and biochemical kits to look for probable differences between the sexes for the liver (ALT, AST, GGT), renal (creatinine and urea), lipid (cholesterol and triglyceride) and protein (albumin and total proteins) profiles. A descriptive statistical analysis was used to calculate the mean, standard deviation, and minimum and maximum values with $95 \%$ confidence. All animals in this study were healthy and hematological and biochemical values for both sexes resembled those of other Callithrix species, including variations between males and females. In addition, the data analyzed can contribute greatly to what is known about this subject for the species in question, especially the serum biochemical values..

Key words: Captivity; Hematology

\section{Introdução}

Algumas espécies de primatas não humanos ( $\mathrm{PNH})$ são utilizadas como modelos biológicos na pesquisa científica. Entre essas espécies incluem-se os saguis-detufo-preto (Callithrix penicillata), pertencentes à família Callitrichinae e que são encontrados em galerias de florestas, extremidades de florestas secundárias, bosques semidecíduos e vegetação de savanas (CHAGAS et al., 1999; VILELA; FARIA, 2004). Podem, ainda, viver em ambientes perturbados e fragmentados uma vez que possuem uma boa adaptabilidade (RYLANDS; FARIA, 1993). Além disso, essa espécie vem sendo criada em diversos biotérios do país (BARINO, 2008).

Essa espécie PNH tem sido modelo experimental para diversas pesquisas científicas, por possuírem características filogenéticas semelhantes às dos seres humanos, e através desses estudos tem sido comprovado seu potencial como hospedeira e transmissora de agentes infecciosos nocivos à saúde humana, sendo, portanto, importante aprofundar os conhecimentos sobre seus padrões biológicos (EPPLE, 1975; SILVA et al., 2014). Callithrix penicillata destacam-se como modelos de estudos pela facilidade de manutenção e sucesso de reprodução em cativeiro, boa adaptação em colônias e em diversos meios, em comparação com espécies de maiores portes (CARVALHO et al., 1991; HIBINO et al., 1999; BARINO et al., 2007).

A participação dos $C$. penicillata em pesquisas envolve estudos relacionados às bacterioses (MELLO et al., 2005), parasitoses (ROSAetal., 2006), viroses (MATZRENSING et al., 2003), toxicidade (HERNÁNDEZ-
LÓPEZ et al., 2005), variações hormonais (MOTA et al., 2006) e comportamento (BURKART; HESCHL, 2007). Os parâmetros hematológicos e bioquímicos são descritos especialmente para saguis mantidos em cativeiro (HAWKEY et al., 1982; MCNEES et al., 1982; YARBROUGH et al., 1984; BOERE et al., 2005; CUNHA et al., 2005; BARBOZA; XAVIER, 2017) e poucos trabalhos estão relacionados a essa temática com animais de vida livre (SILVA et al., 2014; GARIGLIO, 2017).

Os padrões hematológicos e bioquímicos são muito utilizados nos experimentos científicos com PHN (BARINO, 2008; SILVA et al., 2014; BARBOZA; XAVIER, 2017). Através do hemograma são determinadas as variações quantitativas e morfológicas dos componentes do sistema sanguíneo, além do fornecimento de dados importantes para o monitoramento e diagnóstico de enfermidades por meio da aplicação de técnicas pouco invasivas e econômicas (CLARK et al., 2009).

Desta maneira, o estudo dos valores hematológicos e bioquímicos de C. penicillata em cativeiro, onde os parâmetros fisiológicos podem ser melhor monitorados, constitui uma ferramenta imprescindível de contribuição para o conhecimento e o uso dessa espécie como modelo biológico.

\section{Material e Métodos}

A área de estudo consistiu no criatório científico situado no Centro Nacional de Primatas (CENP), o 
qual foi instituído com a Fundação de Serviço de Saúde Pública (F.SESP) pela portaria no $115 / \mathrm{BSB}$, de 15 de março de 1978. Localizado no município de Ananindeua, estado do Pará, BR-316 Km 7, possui uma área de 25 ha e seu plantel é constituído por 21 espécies e subespécies de primatas neotropicais. O município está a uma latitude $01^{\circ} 21^{\prime} 56^{\prime \prime}$ ao sul e a uma longitude $48^{\circ} 22^{\prime} 20^{\prime \prime}$ a oeste. O clima é tropical, úmido, com temperatura elevada, em torno de $25^{\circ} \mathrm{C}$. A pluviosidade encontra-se entre 2.250 e $2.500 \mathrm{~mm}$ com chuvas regulares, com maior concentração de janeiro a junho.

Foram colhidas amostras de sangue de 14 indivíduos (10 machos e quatro fêmeas) adultos da espécie C. penicillata. Os animais foram contidos fisicamente com auxílio de puçás para posterior administração intramuscular de $3,9 \mathrm{mg} / \mathrm{kg}$ da associação de Tiletamina e Zolazepam (Zoletil 50C). Parâmetros fisiológicos como frequência cardíaca e respiratória, reflexos oculares e temperatura foram monitorados até o retorno da anestesia.

O sangue foi colhido por punção venosa utilizandose seringas descartáveis sem anticoagulante, armazenado em tubos e acondicionado em isopor com gelo e imediatamente encaminhado ao Laboratório de Reprodução Animal (LARA) da Universidade Federal do Pará.

Os valores hematológicos foram determinados utilizando-se o contador eletrônico (CELM - CC 500®) e o diluidor eletrônico (CELM DA - 500®). A contagem diferencial de leucócitos foi realizada a partir de leitura com auxílio de microscópio óptico (1000 X) de esfregaço sanguíneo em lâmina corada pelo método Giemsa. A análise incluiu o número de hemácias, hematócrito, hemoglobina, volume corpuscular médio (MCV), hemoglobina corpuscular média $(\mathrm{MCH})$, concentração de hemoglobina corpuscular média (MCHC), linfócitos, leucócitos, neutrófilos segmentados, bastões, eosinófilos, basófilos e monócitos.

O soro foi obtido por centrifugação. As dosagens bioquímicas foram realizadas em duplicata com a utilização de kits comerciais de reativos secos (VITROS DT®) e submetidos aos analisadores (DTSC//Module ${ }^{\circledR}$, DT 60//® e DTE//Module $\left.{ }^{\circledR}\right)$. O método para a realização das dosagens seguiu o padrão estabelecido pelo kit. As dosagens incluíram glicose, colesterol, ureia, triglicerídeos, creatinina, bilirrubina, alanina amino transferase (AST) e transaminase glutâmico pirúvica (ALT), lactato, ácido úrico, amônia, proteínas totais, albumina, soroglobulina, creatina e gamaglutamiltransferase (GGT).

Os dados foram tabulados e analisados mediante estatística descritiva, como média aritmética, desvio padrão, valor mínimo e máximo e os intervalos de 95\% de confiança para cada variável hematológica e bioquímica. Todos os animais foram submetidos e aprovados pela Comissão de Ética no Uso de Animais (CEUA/IEC) do Instituto Evandro Chagas, sob o protocolo de número 07/2016.

\section{Resultados}

Os valores encontrados neste estudo, de maneira geral, podem ser comparados com outros valores da hematologia de saguis descritos na literatura, como os achados de Silva et al. (2014), que foram os primeiros a descrever os valores da hemoglobina no sangue dos saguis híbridos livres na natureza, e Barboza e Xavier (2017), que utilizaram um analisador hematológico com valores de referência padronizados para a espécie C. penicillata.

Os dados dos valores médios hematológicos dos C. penicillata por sexo deste estudo (hemograma e leucograma) são apresentados nas Tabelas 1 e 2.

Os valores dos leucogramas não evidenciaram quadros de leucocitose ou leucopenia, conforme dados da literatura para a espécie $C$. penicillata. Tais valores estão demonstrados na Tabela 2.

Foi realizada a mensuração dos índices bioquímicos (Tabela 3), e constatou-se que os valores bioquímicos séricos para a espécie $C$. penicillata ainda são escassos na literatura, portanto os parâmetros para avaliação e comparação foram baseados no que se encontra descrito para outras espécies de primatas não humanos, principalmente para as espécies do gênero Callithrix. 
TABELA 1: Média, desvio padrão e limites inferiores e superiores do eritrograma de machos e fêmeas de Callithrix penicillata cativos no Centro Nacional de Primatas - CENP, Pará.

\begin{tabular}{lcccccc}
\hline \multirow{2}{*}{ Parâmetro } & \multicolumn{3}{c}{ Machos } & \multicolumn{2}{c}{ Fêmeas } \\
\cline { 2 - 7 } \multicolumn{1}{c}{ N } & Média $\pm \mathbf{D P}$ & $\mathbf{I C ~ 9 5 \%}$ & $\mathbf{N}$ & Média \pm DP & IC 95\% \\
\hline Hematócrito $(\%)$ & 10 & $40,35 \pm 7,84$ & $34,74-45,96$ & 14 & $36,15 \pm 7,13$ & $24,80-47,50$ \\
Hemácias $\left(\mathrm{x} 10^{6} / \mathrm{mm}^{3}\right)$ & 10 & $5,53 \pm 1,13$ & $4,73-6,34$ & 14 & $5,08 \pm 1,18$ & $3,20-6,96$ \\
Hemoglobina $(\mathrm{g} / \mathrm{dl})$ & 10 & $12,00 \pm 2,40$ & $10,28-13,72$ & 14 & $10,96 \pm 2,49$ & $7,01-10,94$ \\
MCV (u $\left.{ }^{3}\right)$ & 10 & $73,04 \pm 4,17$ & $70,06-76,02$ & 14 & $71,80 \pm 4,22$ & $65,09-78,51$ \\
MCH (uug) & 10 & $21,70 \pm 1,42$ & $20,69-22,71$ & 14 & $21,68 \pm 1,02$ & $20,05-23,30$ \\
MCHC (\%) & 10 & $29,70 \pm 1,07$ & $28,94-30,46$ & 14 & $30,20 \pm 1,42$ & $27,95-32,45$ \\
\hline
\end{tabular}

TABELA 2: Média, desvio padrão e limites inferiores e superiores do leucograma de machos e fêmeas de Callithrix penicillata cativos no Centro Nacional de Primatas - CENP, Pará.

\begin{tabular}{lcccccc}
\hline \multirow{2}{*}{\multicolumn{1}{c}{ Parâmetro }} & \multicolumn{2}{c}{ Machos } & \multicolumn{2}{c}{ Fêmeas } \\
\cline { 2 - 7 } & $\mathbf{N}$ & Média \pm DP & IC 95\% & N & Média \pm DP & IC 95\% \\
\hline Leucócitos (x10 $\left./ \mathrm{mm}^{3}\right)$ & 10 & $6,00 \pm 1,94$ & $4,61-7,39$ & 14 & $4,75 \pm 1,50$ & $2,36-7,14$ \\
Basófilo (\%) & 10 & 0 & 0 & 14 & $0,75 \pm 1,50$ & $1,64-3,14$ \\
Eosinófilo (\%) & 10 & $1,20 \pm 0,42$ & $0,9-1,50$ & 14 & $1,25 \pm 0,50$ & $0,45-2,50$ \\
Mielócito (\%) & 10 & 0 & 0 & 14 & 0 & 0 \\
Bastões & 10 & 0 & 0 & 14 & 0 & 0 \\
Segmentados (\%) & 10 & $37,90 \pm 9,65$ & $30,99 \pm 44,81$ & 14 & $50,75 \pm 2,99$ & $46,00-55,50$ \\
Linfócitos (\%) & 10 & $60,40 \pm 10,11$ & $53,17-67,63$ & 14 & $46,25 \pm 1,71$ & $43,53-48,97$ \\
Monócitos (\%) & 10 & $0,50 \pm 0,71$ & $0,01-1,01$ & 14 & $1,00 \pm 0,82$ & $0,30-2,30$ \\
\hline
\end{tabular}

TABELA 3: Média, desvio padrão e limites inferiores e superiores dos parâmetros bioquímicos de machos e fêmeas de Callithrix penicillata cativos no Centro Nacional de Primatas - CENP, Pará.

\begin{tabular}{lcccccc}
\hline \multirow{2}{*}{ Parâmetro } & \multicolumn{4}{c}{ Machos } & \multicolumn{2}{c}{ Fêmeas } \\
\cline { 2 - 7 } & $\mathbf{N}$ & Média \pm DP & IC 95\% & N & Média \pm DP & IC 95\% \\
\hline Glicose (mg/dL) & 10 & $123,80 \pm 38,31$ & $96,4-151,20$ & 14 & $113,75 \pm 8,96$ & $99,50-128,00$ \\
Ureia (mg/dL) & 10 & $11,10 \pm 5,20$ & $7,38-14,82$ & 14 & $14,50 \pm 5,92$ & $5,09-23,91$ \\
Colesterol (mg/dL) & 10 & $117,60 \pm 51,76$ & $80,58-154,62$ & 14 & $83,50 \pm 23,81$ & $41,62-121,38$ \\
Triglicerídeos (mg/dL) & 10 & $123,80 \pm 58,96$ & $81,63-165,97$ & 14 & $79,00 \pm 77,05$ & $43,59-201,59$ \\
Trig. (VLDL) (mg/dL) & 10 & $24,00 \pm 11,88$ & $15,50-32,50$ & 14 & $16,00 \pm 15,43$ & $8,58-40,58$ \\
Creatinina (mg/dL) & 10 & $0,39 \pm 0,07$ & $0,34-0,44$ & 14 & $0,35 \pm 0,06$ & $0,26-0,44$ \\
Bilir. Total (mg/dL) & 10 & $0,85 \pm 0,16$ & $0,74-0,96$ & 14 & $0,76 \pm 0,09$ & $0,62-0,93$ \\
ALT (UI/L) & 10 & - & - & 14 & - & - \\
AST (UI/L) & 10 & $128,90 \pm 123,96$ & $38,80-219,00$ & 14 & $87,50 \pm 31,98$ & $36,61-138,39$ \\
Lactato (mmol/L) & 10 & $25,36 \pm 6,84$ & $20,47-30,25$ & 14 & $18,90 \pm 10,30$ & $2,51-35,29$ \\
Ácido úrico (mg/dL) & 8 & $0,41 \pm 0,45$ & $0,03-0,79$ & 14 & $0,28 \pm 0,55$ & $0,60-1,15$ \\
Amônia (mg/dL) & 10 & $303,10 \pm 248,62$ & $125,26-480,94$ & 14 & $256,75 \pm 124,07$ & $59,35-454,15$ \\
Proteínas totais (g/dL) & 10 & $7,69 \pm 0,96$ & $7,00-8,37$ & 14 & $7,18 \pm 1,32$ & $5,07-9,28$ \\
Albumina (g/dl) & 10 & $4,17 \pm 1,04$ & $3,42-4,92$ & 14 & $3,88 \pm 1,54$ & $1,41-6,34$ \\
Soro/globulina (g/ dl) & 10 & $3,52 \pm 0,55$ & $3,13-3,91$ & 14 & $3,30 \pm 0,32$ & $2,80-3,80$ \\
Creatinina (mg/dl) & 10 & $741,60 \pm 1,65$ & $738,97-744,23$ & 14 & $434,25 \pm 427,26$ & $245,52-114,02$ \\
GGT (UI/ l) & 9 & - & - & 14 & $3,5 \pm 7,00$ & $7,64-14,64$ \\
CKMB (UI/ l) & 10 & $85,90 \pm 140,92$ & $14,90-186,70$ & 14 & $76,75 \pm 20,04$ & $44,87-108,63$ \\
\hline
\end{tabular}




\section{Discussão}

De acordo com os relatos de Birgel (1982), Robel et al. (1996) e Barboza e Xavier (2017), os dados da hematologia para $C$. penicillata, na literatura, podem apresentar conflitos e isso diz respeito à capacidade dessa espécie de adaptabilidade a diferentes ambientes. Isso pode implicar mudanças dos padrões fisiológicos na mesma espécie e na mesma região ou em regiões distintas.

Desse modo, neste estudo, evidenciaram-se valores do eritrograma, a saber, hematócrito, hemácias e hemoglobina, superiores aos encontrados em geral na literatura para esta mesma espécie, tanto em machos como em fêmeas. Tais dados sugerem uma eritrocitose nos animais, a qual pode não ser verdadeira devido às alterações nos padrões fisiológicos dos saguis adaptados aos ambientes de cativeiro, níveis de estresse do ambiente e ao acometimento de parasitoses, conforme relatado na literatura (RAMOS et al., 2004; BARINO, 2008; SILVA et al., 2014; GARIGLIO, 2017).

Em espécies com dimorfismo sexual evidenciamse diferenças hematológicas entre machos e fêmeas devido às especificidades encontradas no status hormonal e na massa muscular entre os gêneros, bem como em espécies que apresentam ciclo menstrual (DE THOISY et al., 2001; WIRZ et al., 2008; MOTSCH et al., 2012). Os valores hematológicos das fêmeas deste estudo foram inferiores aos das fêmeas que não estavam no período gestacional estudadas por Diniz (1997), Peters et al. (2003), Boere et al. (2005) e Barino et al. (2007) e inferiores também aos valores de eritrócitos, hemoglobina e hematócrito dos machos desses estudos. De acordo com Riviello e Wirz (2001), tal fato se deve aos fatores ocasionados pela perda sanguínea no período de menstruação das fêmeas, bem como às deficiências nutricionais e hormonais das mesmas.

Os resultados do hemograma obtidos neste estudo são inferiores aos encontrados em outras espécies de PNH como Cebus sp. (LIMA et al., 2014; FAVARETO et al., 2016; MONTEIRO et al., 2016) e Macaca fascicularis (BONFATI et al., 2009; CYSNE et al., 2015), para ambos os sexos; e ainda esses mesmos resultados mostraram-se diferentes também em relação a outras espécies de saguis, como demonstrado nos trabalhos de Silva et al. (2014), Barboza e Xavier (2017) e Gariglio (2017), sendo que nesses últimos tais valores foram superiores.

Foram observados valores hematimétricos de normocitose e normocromia (MCV, $\mathrm{MCH}$ e $\mathrm{MCHC}$ ) em consonância com os valores hematológicos de referências bibliográficas para animais de cativeiro, enfatizando-se C. penicillata (machos e fêmeas) (BARINO, 2008; BARBOZA; XAVIER, 2017). Em comparação com os valores hematimétricos de $C$. geoffroyi de vida livre, estudados por Gariglio (2017), os valores de MCV e $\mathrm{MCHC}$ neste estudo foram bem menores.

Comparativamente com os relatos da literatura, os valores do MCV e MCHC das fêmeas deste estudo foram superiores aos achados de Diniz (1997) e o mesmo ocorreu com Barino (2008). Porém, a metodologia utilizada para as análises hematológicas foi diferente, uma vez que, neste trabalho e nos de Peters et al. (2003), Boere et al. (2005) e Barino (2008), tais análises foram realizadas por meio de aparelho de automação hematológica, enquanto Diniz (1997) realizou o cálculo manual.

Os autores Failace e Pranke (2004) ressaltaram que os aparelhos automatizados oferecem alta precisão e sensibilidade na realização do hemograma, e esse fato aliado às mudanças estruturais no ambiente de cativeiro explicam porque neste estudo os valores hematimétricos se aproximaram mais aos dos achados dos autores que utilizaram a mesma metodologia de análise através de contadores específicos.

Os valores de leucócitos totais dos animais de C. penicillata em cativeiro foram superiores quando comparados com outras espécies de $C$. penicillata e C.geoffroyi de vida livre estudadas por Silva et al. (2014). Esses valores contradizem o que foi explicitado por Hassimoto et al. (2004) e Flaiban et al. (2008), pois ambos afirmaram que os animais de vida livre estariam mais propensos a apresentar maiores valores de leucócitos totais, devido ao medo da captura e da aproximação do homem.

Os valores dos leucogramas dos machos em relação aos das fêmeas, referentes à apresentação dos valores 
das médias obtidas, foram maiores para leucócitos e linfócitos, principalmente, e menores para segmentados, monócitos e eosinófilos. O mesmo ocorreu com os achados de Nascimento et al. (1993), que estudaram outras espécies de saguis, e com os de Barboza e Xavier (2017) para os linfócitos de C. penicillata. No entanto, em relação aos neutrófilos segmentados, tais valores divergiram quando comparados com estes últimos autores, pois neste estudo foram discrepantes e superiores os das fêmeas. Isso pode ser o indicativo de que o organismo de algumas fêmeas estava lutando contra infecções antigas.

Neste trabalho, os basófilos foram encontrados discretamente somente nas fêmeas, e, nas pesquisas de Silva et al. (2014) e Barboza e Xavier (2017), não foram observados basófilos, em ambos os sexos de C. penicillata. De modo geral, para outras espécies de saguis, não foram encontrados basófilos ou foram discretamente existentes somente nas fêmeas (CUNHA et al., 2005; BARINO, 2008; BARBOSA; XAVIER, 2017; GARIGLIO, 2017).

Na determinação do perfil hepático, os valores de AST foram mais expressivos nos machos e os de CGT foram determinados somente nas fêmeas. $\mathrm{O}$ mesmo ocorreu com a espécie de C. jacchus estudada por Gariglio (2017). Na literatura há divergências entre os resultados encontrados, a exemplo de Silva et al. (2014), em que os valores de AST foram praticamente similares entre machos e fêmeas, apesar de que neste os animais estudados foram de vida livre. Campbell (2006) afirma que, no tecido hepático de primatas, a atividade sérica da ALT aumenta com lesão celular, enquanto a atividade sérica elevada de AST ocorre somente com morte celular, ou seja, o aumento de sua atividade sérica é indicativo de lesões hepatocelulares mais graves, o que não foi evidenciado no presente estudo.

Os valores da albumina e das proteínas totais relacionados ao perfil proteico foram similares entre os machos e as fêmeas de C. penicillata, e tais valores não diferiram dos demais encontrados na literatura para saguis (SILVA et al., 2014; GARIGLIO, 2017) e para outras espécies de PNH (ROSNER et al., 1986; RIVIELLO; WIRZ, 2001; WIRZ et al., 2008; CYSNE et al., 2015; FAVARETO et al., 2016).
Os valores de globulina para os machos e fêmeas de C. penicillata foram similares e fazem parte de um importante grupo de proteínas sintetizadas no fígado e nos plasmócitos, estando relacionadas a diferentes funções, tais como transporte e defesa, de acordo com Gariglio (2017). Para saguis híbridos já foram encontrados valores maiores de globulinas em fêmeas (SILVA et al., 2014).

Referente aos parâmetros renais analisados, os valores de ureia para as fêmeas em relação aos machos foram maiores, e o contrário ocorreu com os valores de creatinina. O mesmo ocorreu com os parâmetros renais de $C$. geoffroyi avaliados por Gariglio (2017). No entanto, para Silva et al. (2014), tanto os valores de ureia quanto os de creatinina foram superiores nos machos em relação às fềmeas para $C$. penicillata e $C$. geoffroyi.

Neste estudo, os níveis de colesterol foram superiores nos machos quando comparados com as fêmeas, tal como já foi descrito na literatura para saguis (CLARKE, 1994; SILVA et al., 2014; GARIGLIO, 2017) e para outras espécies de PNH (ROSNER et al., 1986; MÁLAGA et al., 1991; SCHMIDT et al., 2007; ROVIROSA-HERNÁNDEZ et al., 2012). Em relação às demais espécies do gênero Callithrix de vida livre e de cativeiro, neste estudo, os níveis de colesterol, triglicerídeos e de glicose do C. penicillata foram inferiores aos valores descritos para tais espécies (MCNEES et al., 1982; YARBROUGH et al., 1984; SILVA et al., 2014; GARIGLIO, 2017).

Os resultados da análise bioquímica obtidos neste estudo não foram similares aos dados encontrados para espécies de cativeiro de $C$. penicillata, C. jaccus e $C$. geoffroyi (YARBROUGH et al., 1984; NASCIMENTO et al., 1993; PETERS et al., 2003; BOERE et al., 2005; SILVA et al., 2014; GARIGLIO, 2017). Além disso, os parâmetros bioquímicos refletem diretamente o status nutricional dos animais, principalmente em relação ao consumo de proteína e carboidratos (SINGLETON et al., 2015; CHOI et al., 2016), a alterações na função renal e hepática ou a diferenças na ingestão de água (THRALL et al., 2007).

Baseados nos resultados, podemos concluir que as mudanças no meio ambiente decorrentes dos impactos 
antrópicos podem influenciar na fisiologia reprodutiva dessa espécie, visto que são submetidas a situações de estresse e mudanças comportamentais. Devido a esses fatos, são encontradas na literatura diferenças nos valores hematológicos e bioquímicos entre as espécies do gênero Callithrix, apesar de algumas similaridades entre elas e da capacidade adaptativa.

Os valores dos parâmetros bioquímicos sanguíneos (proteínas totais, albumina, globulina, glicose e colesterol), assim como os valores dos parâmetros que indicam funções renais e hepáticas (ureia, creatinina, AST e ALT), neste estudo, diferiram entre machos e fêmeas, porém com algumas semelhanças em comparação aos poucos trabalhos com primatas não humanos do gênero Callithrix.

Essas informações elucidadas podem contribuir para indicação de valores de referência para a espécie, os quais são considerados pela comunidade científica como pontos críticos na implementação de programas de manejo e conservação do gênero Callithrix.

\section{Agradecimentos}

Os autores agradecem ao Centro Nacional de Primatas-CENP e à Universidade Federal do Pará-UFPA pela colaboração, idealização e execução desta pesquisa.

\section{Referências}

BARBoZA, J. M. O. C.; XAVIER, K. V. M. Parâmetros hematológicos de Callithrix jacchus linnaeus, 1758 e Callithrix penicillata Étienne Geoffroy, 1812, do Centro de Manejo de Fauna da Caatinga - CEMAFAUNA - caatinga, no município de Petrolina, Pernambuco. II. 2017. In: CONGRESSO INTERNACIONAL DA DIVERSIDADE DO SEMI-ÁRIDO, Campina Grande. Anais... Vol. 1, Campina Grande: CONIDIS, 2017. Disponível em: <http:// www.cemafauna. univasf.edu.br/pagina_simples.php?pagina $=$ resu mos\&titulo $=$ Resumos $>$.

BARINO, G. T. M. Padrão hematológico de fêmeas de Callithrix penicillata, Étienne Geoffroy, 1812 (Primates: Cebidae). 2018. 82 f. Dissertação (Mestrado em Ciências Biológicas) - Universidade Federal de Juiz de Fora, Juiz de Fora. 2008.

BARINO, G. T. M.; PINTO, R. M.; GUERRA, M. O.; PETTERS, V. M.; ARAÚJO, D. A. C. Perfil reprodutivo do Callithrix penicillata em cativeiro, In: BICCA-MARQUES, J. C. (Ed). A Primatologia do Brasil. 10 ed. Porto Alegre: Sociedade Brasileira de Primatologia, 2007. p. 511-519.
BIRGEL, E. H. Hematologia clínica veterinária, In: BIRGEL, E. H.; BENESE, F. J. (Ed.). Patologia Clínica Veterinária. São Paulo. Sociedade Brasileira de Medicina Veterinária, 1982. p. 2-34. BOERE, V.; PINHEIRO, E. C.; DE OLIVEIRA, S. I.; PALUDO, G. R.; CANALE, G.; PIANTA, T.; WELKER, A.; ROCHADE-MOURA, R. C. Comparison between sex and age class on some physiological, thermal, and hematological indices of the cerrado's marmoset (Callithrix penicillata). Journal of Medical Primatology, Copenhagen, v. 34, n. 3, p. 156-162, 2005.

BONFATI, U.; LAMPARELLI, D.; COLOMBO, P.; BERNARDI, C. Hematology and serum chemistry parameters in juvenile cynomolgus (Macaca fascicularis) of Mauritius origin: comparison between purpose-bred and captured animals. Journal of Medical Primatology, Copenhagen, v. 38, p. 228-235, 2009.

BURKART, J. M.; HESCHL, A. Understanding visual access in common marmosets Callithrix jacchus: perspective taking or behaviour reading? Animal Behavior, Bloomington, v. 73, n. 3, p. 457-469, 2007.

CAMPBELL, T.W. Hematologia de mamíferos: animais de laboratório e espécies variadas. In: THRALL, M. A.; WEISER, G.; ALLISON, R. W.; CAMPBELL, T. W. (Ed.). Hematologia e Bioquímica Clínica Veterinária. Cap.16. 2. ed. Rio de Janeiro: Editora Roca, Guanabara Koogan, 2006. p. 201-214.

CARVAlHO, A. C. T.; CISALPINO, P. S.; CISALPINO, E. O.; PEREIRA, L. H.; MELO, A. L. Estudos da microbiota intestinal do mico-estrela (Callithrix penicillata) em diferentes estágios de lactação. In: RYLANDS, A. B.; BERNARDES A. T. (Ed.), A primatologia do Brasil. 3 ed. Belo Horizonte: Sociedade Brasileira de Primatologia, 1991. p. 103-110.

CHAGAS, A. C. S.; PIEMONTE, D. V.; RAPOSO-FILHO, J. Observações comportamentais de Callithrix penicillata (Geoffroy, 1992) (Callitrichinae: Primates) na Reserva Biológica Santa Cândida em Juiz de Fora, Minas Gerais. Bioscience Journal, Uberlândia, v. 15, n. 2, p. 25-33, 1999.

CHOI, K.; CHANG, J.; LEE, M. J.; WANG, S.; IN, K.; GALANOTAN, W. C.; JUN, S.; CHO, K.; HWANG, Y. H.; KIM, S. J.; PARK, W. Reference values of hematology, biochemistry, and blood type in cynomolgus monkeys from Cambodia origin. Laboratory Animal Research, Seoul, v. 32, n. 1, p. 46-55, 2016.

CLARK, P.; BOARDMAN, W. S. J.; RAIDAL, S. Collection and handling of blood samples. 2009. Disponível: $<$ https://veteriankey. com/collection-and-handling-of-blood-samples/ $>$.

CLARKE, J. M. The common marmoset (Callithrix jacchus). Anzccart News, Adelaide, v. 7, p. 1-8, 1994.

CUNHA, M. S.; LOPES, D. R.; SOUSA, M. B. C. Variação na contagem de leucócitos do Callithrix jacchus (Linnaeus, 1758) submetidos a uma situação de estresse agudo. Revista Brasileira de Zoociências, Juiz de Fora, v. 7, n. 2, p. 217-229, 2005.

CYSNE, L. B.; ANDRADE, M. C. R.; GONÇALVES, M. A. B.; LOPES, C. A. A.; RAMOS, S.; ZANINI, G. M.; CABELLO, P. H. Avaliação clínica, morfométrica e hematológica de macacos Cynomolgus (Macaca fascicularis) cativos. RESBICAL, São Paulo, v. 3, n. 1, p. 36-44, 2015.

DE THOISY, B.; VOGEL, I.; REYNES, J. M.; POULIQUEN, J. F.; CARME, B.; KAZANJI, M.; VIÉ, J. C. Health evaluation of 
translocated free-ranging primates in French Guiana. American Journal of Primatology, New York, v. 54, n. 1, p. 1-16, 2001.

DINIZ, L. S. M. Primatas em cativeiro - Manejo e problemas Veterinários. São Paulo: Ícone Editora, 1997. 196 p.

EPPLE, G. The behavior of marmoset monkeys (Callitrichidae). In: ROSEMBLUM, L. A. (Ed.). Primate Behavior. New York: Academic Press, 1975. p. 195-239.

FAILACE, R.; PRANKE, P. Avaliação dos critérios de liberação direta dos resultados de hemogramas através de contadores eletrônicos. Revista Brasileira de Hematologia e Hemoterapia, São José do Rio Preto, v. 26, n. 3, p. 159-166, 2004.

FAVARETO, A. D.; COSTA, M. M.; GRUMANN, M. R.; MOTTA, A. C.; SILVA-FILHO, J. R.; SILVA, Z.; DUDA, N. C.; NUNES, N. J.; VALLE, S. F. Perfil hematológico e bioquímico de macacospergo (Cebus spp.) mantidos em cativeiro. Revista Brasileira de Medicina Veterinária, Rio de Janeiro, v. 38, n. 4, p. 406-412, 2016.

FLAIBAN, K. K. M. C.; SPOHR, K. A. H.; MALANSKI, L. S.; SVOBODA, W. K.; SHIOZAWA, M. M.; HILST, C. L. S.; AGUIAR, L. M.; PASSOS, F. C.; NAVARRO, I. T.; LISBOA, J. A. N.; BALARIN, M. R. S. Valores hematológicos de bugios pretos (Alouatta caraya) de vida livre da região do Alto Rio Paraná sul do Brasil. Arquivo Brasileiro de Medicina Veterinária e Zootecnia, Belo Horizonte, v. 61, p. 628-634, 2008.

GARIGLIO, A. C. R. S. Valores hematológicos e bioquímico sanguíneo de Callithrix geoffroyi sob influência antrópica no estado do Espírito Santo. 2017. 33 f. Dissertação (Mestrado em Biologia Animal) - Universidade Federal de Viçosa, Viçosa. 2017.

HASSIMOTO, M.; HARRADA, T.; HARRADA, T. Changes in hematology, biochemical values, and restraint ECG of rhesus monkeys (Macaca mulatta) following 6-month laboratory acclimation. Journal of Medical Primatology, Copenhagen, v. 33, p. $175-186,2004$

HAWKEY, C. M.; HART, M. G.; JONES, D. M. Clinical hematology of the common marmoset Callithrix jacchus. American Journal of Primatology, New York, v. 3, n. 1-4, p. 179-199, 1982.

HERNÁNDEZ-LÓPEZ, L.; UMLAND, N.; MONDRÁGONCEBALLOS, L.; NAYUDU, P. L. Comparison of the effects of Percoll and Puresperm ${ }^{\circledR}$ on the common marmosets (Callithrix jacchus) semem. Journal of Medical Primatology, Copenhagen, v. 34, n. 2, p. 86-90, 2005.

HIBINO, H.; TONY, K.; IKEBUCK, K.; WU, M. S.; SUGIYAMA, H.; NAKAZAKI, Y.; TANABE, T.; TAKAHASHI, S.; TOJO, A.; SUZUKI, S.; TANIOKA, Y.; SUGIMOTO, Y.; NAKAHATA, T.; ASANO, S. The common marmoset as target preclinical primate model for cytokina and gene therapy studies. Blood, Washington, v. 93, n. 9, p. 2839-2848, 1999.

LIMA, C. B. D.; SANTOS, K. M. M.; ALMEIDA, H. M.; NASCIMENTO, C. B., JÚNIOR, A. M. C.; RIZZO, M. S. Avaliação do perfil hematológico, bioquímico e esfregaço de sangue periférico com vistas ao perfil sanitário em primatas do gênero Cebus mantidos em cativeiro. Semina: Ciências Agrárias, Londrina, v. 35, p. 1847-1854, 2014.

MÁlAGA, C. A.; WELLER, R. E.; MONTOYA, E.; MORO, J.; BUSCHBOM, R. L. Mortality and body weight changes in Aotus nancymai shipped from Iquitos, Peru to Richland, Washington.
Journal of Medical Primatology, Copenhagen, v. 20, n. 1, p. 6-11, 1991.

MATZ-RENSING, K.; JENTISCH, K. D.; LANGENHUYZEN, F.; VERSCHOOR, E.; NIPHUIS, H.; KAUP, F. J. Fatal herpes simples infection in a group of common marmosets (Callithrix jacchus). Veterinary Pathology, Seattle, v. 40, p. 405-411, 2003.

MCNEES, D. W.; PONZIO, B. J.; LEWIS, R. W.; STEIN, F. J.; SIS, R. F.; LEVY, B. M. Hematology of common marmosets (Callithrix jacchus). Primates, Kyoto, v. 23, n. 1, p. 145-150, 1982.

MELlO, M. F. V.; MONTEIRO, A. B. S.; PISSINATI, A.; FERREIRA, A. M. Identification of Helicobacter sp. in gastric mucosa from captive marmosets (Callithrix sp., Callitrichidae, Primatas). American Journal of Primatology, New York, v. 66, n. 2, p. 111-118, 2005.

MONTEIRO, F. O. B.; MONTEIRO, M. V. B.; SCOFIELD, A.; WHITEMAN, C. W.; A. F. ALFIERI, A.; ALFIERI, A. A. Hematological and biochemistry evaluation in capuchin monkeys from the illegal captivity. Acta Veterinaria Brasilica, Mossoró, v. 10, n. 1, p. 92-97, 2016.

MOTA, M. T. S.; FRANÇA, C. R.; CORDEIRO, M. B. S. Hormonal changes related to paternal and alloparental care in common marmosets (Callithrix jacchus). Hormones and Behavior, Ithaca, v. 49, n. 3, p. 293-302, 2006.

MOTSCH, P.; GONZALEZ, J. P.; VERRIER, D. Clinical biochemistry and hematology of the elusive sun-tailed monkey (Cercopithecus solatus) in Gabon: inaugural data from the only semifree ranging colony in the world. American Journal of Primatology, New York, v. 74, n. 3, p. 236-246, 2012.

NASCIMENTO, M. D.; PISSINATTI, A.; CRUZ; J. B.; COIMBRA-FILHO, A. F. Hematological profiles of Callithrix geoffroyi (Humboldt, 1812), Callithrix kuhli (Wied, 1826) and Callithrix aurita (Geoffroyi, 1812). (Callitrichidae - Primates). In: YAMAMOTO, M. A.; SOUZA, M. B. C. (Org.). A Primatologia no Brasil. Belo Horizonte: Sociedade Brasileira de Primatologia, 1993. p. 227-242.

PETERS, V. M.; LESSA, W. D. D. A.; GUERRA, M. O. Callithrix jacchus penicillata blood count. Boletim do Centro de Biologia de Reprodução, Juiz de Fora, v. 22, p. 101-104, 2003.

RAMOS, T. M. B.; VASCONCELOS, A. S.; CARVALHO, V. C. O.; LIMA, V. L. M. Alterações nos níveis de colesterol, triglicerídeos e fosfolipídeo total em plasma de Callithrix jacchus (sagui) reinfectado por Schistosoma mansoni. Revista da Sociedade Brasileira de Medicina Tropical, São Paulo, v. 37, p. 37-40, 2004. RIVIELLO, M. C.; WIRZ, A. Hematology and blood chemistry of Cebus apella in relation to sex and age. Journal of Medical Primatology, Copenhagen, v. 30, p. 308-312, 2001.

ROBEL, G. L.; LOCHMILLER, R. L.; MCMURRY, S. T.; QUALIS-JR, C. W. Environmental, age and sex effects on cotton rat (Sigmodon spidus) hematology. Journal of Wildlife Diseases, Atlanta, v. 32, n. 2, p. 390-394, 1996.

ROSA, D. S.; IWAI, L. K.; TZELEPIS, F.; BARGIERI, D. Y.; MEDEIROS, M. A.; SOARES, I. S.; SIDNEY, J.; SETTE, A.; KALIL, J.; MELLO, M. E.; CUNHA-NETO, E.; RODRIGUES, M. M. Immunogenicity of recombinant protein containing the Plasmodium vivax vaccine candidate $\mathrm{MSP}_{19}$ and two human $\mathrm{CD} 4^{+}$ T-cell epitopes administered to no human primates (Callithrix 
jacchus jacchus). Microbes and Infection, Sacramento, v. 8, n. 8 , p. 2130-2137, 2006.

ROSNER, J. M.; SCHININI, A.; ROVIRA, T.; MERLO, R.; BESTARD, R.; MALDONADO, M. Body measurements, hematology, and serum chemistry values of the adult Cebus apella monkey. Journal of Medical Primatology, Copenhagen, v. 15, n. 4, p. 295-302, 1986.

ROVIROSA-HERNÁNDEZ， M. J.; CABA， M.; GARCÍAORDUÑA, F.; LÓPEZ-MUÑOZ, J. J. D.; CANALES-ESPINOSA, D.; HERMIDA-LAGUNES, J. Hematological and biochemical blood values in wild populations of black howler monkeys (Alouatta pigra) of Campeche, México. Journal of Medical Primatology, Copenhagen, v. 41, n. 5, p. 309-31, 2012.

RYLANDS, A. B.; FARIA, D. S. Habitats, feeding, and home range size in the genus Callithrix, In: RYLANDS, A. B. (Ed.), Marmosets and Tamarins: systematics, behaviour and ecology. New York: Oxford University Press, 1993. p. 262-272.

SCHMIDT, D. A.; KOWALEWSKI, M. M.; ELLERSIECK, M. R.; ZUNINO, G. E.; STACEWICZ-SAPUNTZAKIS, M.; CHEN, T. C.; HOLICK, M. F. Serum nutritional profiles of free-ranging Alouatta Caraya in Northern Argentina: lipoproteins, amino acids, vitamins $\mathrm{A}, \mathrm{D}$, and $\mathrm{E}$, carotenoids, and minerals. International Journal of Primatology, New York, v. 28, n. 5, p. 1093-1110, 2007. SILVA, I. O.; SILVA, F. F. R.; FUZESSY, L. F.; TAVELA, A. O.; JÚNIOR, M. C.; SILVA, V. H. D.; PAULA, T. A. R., BOERE, V. Hematology and blood biochemistry in wild hybrid marmosets from the Atlantic Forest, Brazil. Ciência Rural, Santa Maria, v. 44, n. 9, p. 1596-1602, 2014.
SINGLETON, C. L.; NORRIS, A. M.; SAUTHER, M. L.; CUOZZO, F. P.; YOUSSOUF JACKY, I. A. Ring-tailed lemur (Lemur catta) health parameters across two habitats with varied levels of human disturbance at the Bez Mahafaly Special Reserve, Madagascar. Folia Primatologica, Basel, v. 86, n. 1-2, p. 56-65, 2015.

THRALL, M. A.; BAKER, D. C.; CAMPBELL, T. W.; DENICOLA, D.; FETTMAN, M. J.; LASSEN, E. D.; REBAR A.; WEISER, G. Interpretação da resposta leucocitária nas doenças, In: IBID (Ed.). Hematologia e Bioquímica Clínica Veterinária. Rocca: São Paulo, 2007. p. 127-140.

VILELA, S. L. E.; FARIA, D. S. Seasonality of the activity pattern of Callithrix penicillata (Primates, Callitrichidae) in the cerrado (scrub savana vegetation). Brazilian Journal of Biology, São Carlos, v. 64, n. 2, p. 363-370, 2004.

WIRZ, A.; TRUPPA, V.; RIVIELLO, M. C. Hematological and plasma biochemical values for captive tufted capuchin monkeys (Cebus apella). American Journal of Primatology, New York, v. 70, n. 5, p. 463-472, 2008.

YARBROUGH, L. W.; TOLLETT, J. L.; MONTREY, R. D.; BEATTIE, R. J. Serum biochemical, hematological and body measurement data for common marmosets (Callithrix jacchus jacchus). Laboratory Animal Science, Cordova, v. 34, n. 3, p. 276-280, 1984. 